\title{
SANEAMIENTO Y DETOXIFICACIÓN DE CARNE DE LLAMA (Lama glama) INFECTADA CON Sarcocystis aucheniae MEDIANTE COCCIÓN, HORNEADO, FRITURA Y CONGELADO
}

\author{
SANITATION AND DETOXIFICATION OF LAMA MEAT (LAMA GLAMA) INFECTED WITH \\ SARCOCYSTIS AUCHENIAE THROUGH BOILING, BAKING, FRYING AND FREEZING \\ Roxana Godoy Z. ${ }^{1}$, Miguel Vilca L. ${ }^{2}$, Armando Gonzáles Z. ${ }^{3}$, \\ Víctor Leyva V. ${ }^{4}$ y Rosa Sam T. ${ }^{5}$
}

\section{Resumen}

Con el objetivo de sanear y detoxificar la carne de llama infectada con Sarcocystis aucheniae se evaluó el efecto de los métodos físicos de cocción $\left(100{ }^{\circ} \mathrm{C}\right.$ por $\left.10 \mathrm{~min}\right)$, horneado $\left(105^{\circ} \mathrm{C}\right.$ por $\left.65 \mathrm{~min}\right)$, fritura y congelado $\left(-20^{\circ} \mathrm{C}\right.$ por 10 días). La inactivación de la toxicidad de la proteína sarcocystina se evaluó en 30 conejos de $4-5$ meses de edad que fueron inoculados s.c. con $100 \mu \mathrm{g}$ de proteína/kg de peso vivo, obtenida de un lisado de macroquistes de $S$. aucheniae proveniente de carnes tratadas con cada método físico y de carne sin tratar. Únicamente los conejos del control positivo (carne sin tratar) murieron. Los conejos del grupo de carne congelada presentaron sintomatología tóxica moderada. La interrupción del ciclo biológico del $S$. aucheniae se evaluó en 13 perros de 2-5 meses de edad que fueron alimentados con $200 \mathrm{~g}$ de carne de llama tratada o sin tratar. Los perros de los grupos de carne tratada no eliminaron esporoquistes en las heces a comparación de los perros del grupo control positivo que los eliminaron a partir de los 14 días de la ingestión de carne. Se concluye que la cocción, horneado y fritura (y la congelación en forma limitada) lograron desnaturalizar y detoxificar la sarcocystina de los macroquistes de Sarcocystis aucheniae; así mismo, los cuatro tratamientos afectaron la viabilidad de los quistes, eliminando el riesgo potencial de infección del hospedero definitivo.

Palabras clave: Sarcocystis aucheniae, macroquistes, esporoquistes, conejos, perros

\section{Abstract}

The aim of the study was the sanitation and disinfecting llama meat naturally infected with macrocysts of Sarcocystis aucheniae through any of four physical methods: boiling (100 ${ }^{\circ} \mathrm{C}$ for $\left.10 \mathrm{~min}\right)$, baking $\left(105^{\circ} \mathrm{C}\right.$ for $\left.65 \mathrm{~min}\right)$, frying and freezing $\left(-20^{\circ} \mathrm{C}\right.$ for 10 days). A lisis of macrocysts from treated and non-treated meats was prepared and inoculated (100 $\mu \mathrm{g} / \mathrm{kg}$ of body weight, subcutaneously).into 30 rabbits of 4-5 months of age. Only rabbits of the positive control group (non-treated meat) died. Rabbits of the frozen meat group

\footnotetext{
${ }^{1}$ Práctica privada

${ }^{2}$ Laboratorio de Salud Pública y Saneamiento Ambiental, FMV-UNMSM

E-mail: mvilcal@unmsm.edu.pe

${ }^{3}$ Laboratorio de Medicina Veterinaria Preventiva, FMV-UNMSM

${ }^{4}$ Laboratorio de Reproducción Animal, FMV-UNMSM

${ }^{5}$ Laboratorio de Microbiología y Parasitología Veterinaria, FMV-UNMSM
} 
showed moderate toxicity signs. Furthermore, 13 puppies (2-5 months of age) were fed with $200 \mathrm{~g}$ of treated or non-treated infected llama meat. Puppies of meat-treated groups did not eliminate sporocysts in the feces as compared to puppies of the non-treated meat that had sporocysts after 14 days of the feeding. It can be concluded that boiling, baking, and frying (and freezing in some extent) cleaned and disinfected the toxin in the macrocysts of $S$. aucheniae and all four methods affected cysts viability.

Key words: Sarcocystis aucheniae, macrocysts, esporocysts, rabbit, dog

\section{INTRODUCCIÓN}

La llama forma la parte minoritaria de rebaños mixtos en el altiplano peruano, donde cumple un rol social como animal de carga, así como un rol productivo proveyendo carne al componente familiar (Leyva, 1991) y en forma limitada al mercado local. La aceptación de la carne de llama por el público consumidor es baja debido a que su calidad se encuentra afectada por la presencia de quistes de Sarcocystis aucheniae en la canal (Guerrero et al., 1967; Castro 1974; Leguía, 1987; Medrano, 2006) y que en muchos casos ocasiona su decomiso con pérdidas de \$300,000.00 dólares anuales (Alva et al., 1980; Leguía, 1991).

Se reportan tres especies de Sarcocystis en los camélidos sudamericanos (CSA): Sarcocystis tilopodi (S. guanicoecanis) en guanacos, Sarcocystis aucheniae en alpacas, llamas y vicuñas, y Sarcocystis lamacanis en llamas y alpacas (Leguía, 1999).

El perro, usado por los productores de camélidos sudamericanos (CSA) en el manejo del rebaño (Cordero del Campillo, 1999), es el hospedero definitivo del Sarcocystis aucheniae (Leguía et al., 1989) y responsable de la latencia e incidencia de la enfermedad. Los perros infectados eliminan millones de esporoquistes en las heces que son depositados en los pastos, donde permanecen viables por meses en condiciones de humedad y bajas temperaturas (Leguía, 1999) constituyendo una fuente de infección para los CSA. En el ciclo biológico de este parásito, el perro representa el hospedero donde los estadios sexuales se desarrollan en el intestino y los CSA el hospedero para el desarrollo del estadio asexual en el endotelio vascular y músculos (Leguía et al., 1989).

En salud pública, el consumo de carne infectada con macroquistes cobra importancia por ocasionar trastornos digestivos, dolor abdominal, diarrea, escalofríos, nauseas y vómitos (Leguía et al., 1989); sin embargo, también se reportan los mismos signos aunque en forma más drástica, por el consumo del corazón infectado con microquistes (Leguía, 1999). El efecto es atribuido a una proteína tóxica (sarcocystina) que se encuentra dentro del quiste (Sam, 1988), que tiene una actividad neurotóxica a nivel de músculo cardíaco y tejido nervioso gastrointestinal (Hiepe et al., 1981).

Un método tradicional que podría permitir eliminar la toxicidad de los quistes de $S$. aucheniae es la preparación de charqui (Leguía y Arévalo, 1990), aunque no hay estudios serios que lo avalen. Por otro lado, Un estudio reciente señala la posibilidad de inactivar la toxina mediante el autoclavado de la carne (Durán, 2004). El presente estudio tuvo como objetivo evaluar métodos físicos que puedan inactivar la toxina proteica de los quistes de S. aucheniae en carne de llama, así como la interrupción del ciclo biológico.

\section{MATERIALeS Y MéTOdos}

\section{Procedimiento experimental}

Se utilizó 15 llamas adultas, beneficiadas, en el camal de Huancavelica, cuyas 
canales estaban infectadas con macroquistes de Sarcocystis. La carne fue fileteada en trozos de $1 \mathrm{~cm}$ de grosor y dividida en cinco porciones, donde se aplicó los siguientes tratamientos:

a) Cocción en agua. La carne fue sumergida en agua caliente hasta alcanzar una temperatura interna de $100^{\circ} \mathrm{C}$ durante 10 minutos.

b) Horneado. La carne fue envuelta en papel metálico e introducida en un horno eléctrico a una temperatura de $105^{\circ} \mathrm{C}$ por 65 minutos hasta lograr su cocción.

c) Fritura. La carne fue frita en aceite caliente hasta lograr su cocción.

d) Congelación. La carne fue envuelta en bolsas plásticas e introducida en una congeladora eléctrica vertical a una temperatura de $-20^{\circ} \mathrm{C}$ por 10 días.

e) Control. Carne sin tratamiento.

El mínimo tamaño muestral de animales por grupo experimental, según la fórmula de Snedecor y Cochran (1989), fue de tres; sin embargo, para la evaluación de la detoxificación de la carne se utilizó 5 conejos por grupo, en tanto que para la evaluación del riesgo potencial de infección del hospedero definitivo se tuvo que utilizar 2 perros en la mayoría de los grupos experimentales. La distribución de los animales en cada grupo fue hecha al azar.

\section{Evaluación de la detoxificación de carne tratada físicamente}

Se extrajeron los macroquistes de las carnes tratadas y no tratadas. Estos fueron suspendidos en solución salina fosfatada (PBS) $0.15 \mathrm{M}$ y con $\mathrm{pH}$ de 7.2, decantados, y luego machacados en un mortero congelado a $-20^{\circ} \mathrm{C}$ hasta obtener una pasta homogénea. Se le agregó $8 \mathrm{ml}$ de PBS y esta solución, conteniendo extractos de bradizoitos de Sarcocystis, fue tamizada con gasa y colocada en un vaso estéril de $10 \mathrm{ml}$. Posteriormente, estas estructuras celulares fueron lisadas por ultrasonido a 60 ciclos/s en 4 intervalos de $60 \mathrm{~s}$, y centrifugadas a 12,000 g por $20 \mathrm{~min}$
(Sam et al., 1998). A la solución resultante se le añadió antibiótico (100 UI de penicilina y $100 \mathrm{mg}$ de estreptomicina), colectándose el sobrenadante en frascos estériles de 10 $\mathrm{ml}$ y congelándose a $-20^{\circ} \mathrm{C}$ hasta su uso. Se midió la concentración de proteínas en un espectofotómetro (Photometro 4010 Manheim Boehringer) a una longitud de onda de $540 \mathrm{~nm}$.

Se utilizó 30 conejos de 4-5 meses de edad, de raza Nueva Zelanda, los cuales se mantuvieron en cuarentena por 30 días antes del inicio del experimento. De estos, 20 conejos (5 por tratamiento) fueron inoculados subcutáneamente con $100 \mu \mathrm{g}$ de proteína/kg peso vivo, provenientes del lisado de macroquistes de Sarcocystis extraídos de carnes tratadas, 5 conejos (control positivo) recibieron los inóculos de carne sin tratar, y 5 (control negativo) fueron inoculados con suero fisiológico.

Se registraron signos clínicos compatibles con intoxicación (postración, disnea, pupila contraída, hipertermia, congestión conjuntival, diarrea y muerte) durante $27 \mathrm{~h}$ post-inoculación (Sam et al., 1998; Durán, 2004; Céspedes, 2005). Los signos se clasificaron como leve (al menos un signo clínico), moderado (más de un signo clínico) y severo (muerte). Se midió la temperatura rectal a la 1, 4, 8 y 16 horas post-inoculación y se registraron las lesiones macroscópicas en órganos de los animales que murieron.

\section{Evaluación del riesgo potencial de in- fección del hospedero definitivo}

Se empleó 13 perros cruzados de 2-5 meses de edad, los cuales fueron desparasitados y vacunados antes del ensayo (Período de cuarentena de 60 días). Todos los animales recibieron alimento comercial de acuerdo a sus necesidades nutricionales y agua ad libitum, y se alojaron en caniles previamente lavados y desinfectados.

Ocho perros recibieron las carnes tratadas (2 por cada tratamiento físico), 3 reci- 
Cuadro 1. Temperatura rectal promedio en conejos inoculados con lisados de macroquistes de Sarcocystis aucheniae provenientes de carnes tratadas por métodos físicos

\begin{tabular}{lccccc}
\hline \multirow{2}{*}{ Tratamiento } & \multicolumn{5}{c}{ Temperatura rectal post-inoculación $\left({ }^{\circ} \mathrm{C}\right)$} \\
\cline { 2 - 6 } & $0 \mathrm{~h}$ & $1 \mathrm{~h}$ & $4 \mathrm{~h}$ & $8 \mathrm{~h}$ & $16 \mathrm{~h}$ \\
\hline Cocción & 38.5 & 38.7 & 40.4 & 40.6 & 39.0 \\
Horneado & 38.4 & 38.6 & 40.8 & 40.4 & 39.7 \\
Fritura & 38.3 & 38.7 & 40.7 & 40.6 & 39.4 \\
Congelación & 38.6 & 39.2 & 41.1 & 40.7 & 40.2 \\
Control positivo & 38.3 & 39.4 & 41.3 & 40.4 & murieron \\
Control negativo & 38.6 & 38.4 & 39 & 39.6 & 38.9 \\
\hline
\end{tabular}

bieron carne sin tratar y dos quedaron como controles recibiendo sólo alimento comercial. Cada perro ingirió $200 \mathrm{~g}$ de carne conteniendo un mínimo de 100 quistes.

Se colectó muestras diarias de heces por 30 días considerando que el periodo prepatente del $S$. aucheniae en perros es de 11 a 20 días (Leguía et al., 1989). Las heces se analizaron por el método de flotación con solución saturada de sal (Soulsby, 1988).

\section{Resultados y Discusión}

Los conejos que recibieron los inóculos provenientes de macroquistes de carnes tratadas por alguno de los cuatro métodos físicos sobrevivieron al efecto de la proteína del Sarcocystis. Así mismo, los perros alimentados con las carnes sometidas a los tratamientos físicos no eliminaron esporoquistes en las heces. Estos resultados indicarían que los métodos físicos usados lograron inactivar la toxina proteica y la viabilidad de los quistes de Sarcocystis, interrumpiendo así, la fase sexual en el hospedero definitivo.

Los cambios de temperatura rectal de los conejos antes y después de recibir el inóculo se muestra en el Cuadro 1. La temperatura en los conejos inoculados con lisados de macroquistes de carnes tratadas se incrementó en las primeras 8 horas post-inoculación, disminuyendo a las 16 horas, con excepción del tratamiento con congelación, aunque manteniendo valores superiores al periodo pre-inoculación. Estos resultados indican que no se logró una desnaturalización completa de la proteína ya que la toxina no quedó inactiva. La hipertermia se atribuye a los agentes desencadenantes llamados pirógenos exógenos, que actúan mediante un pirógeno endógeno idéntico a la interleucina 1 (IL-1). Este pirógeno, al ponerse en contacto con las neuronas del área preóptica del hipotálamo, actúa sobre el termostato elevando su nivel (Guinart y López, 1997).

Los conejos del grupo de congelación mostraron una moderada sintomatología tóxica, con hipertermia, depresión, congestión de conjuntiva y anorexia, indicando una incompleta acción detoxificante de la proteína del quiste. Estos signos disminuyeron en el transcurso del experimento y fueron menos marcados que los presentados en el grupo control positivo.

En el grupo control positivo se observó anorexia, depresión, postración, conjuntiva ocular congestionada, lagrimeo, heces líquidas, ataxia, opistótonos y muerte, demostrándose así el efecto letal de la toxina. Las muer- 
tes ocurrieron entre las 8 y 11 horas de la inoculación. Estos signos coinciden con los efectos letales reportados por Sam et al. (1998), Durán (2004) y Céspedes (2005).

En la necropsia se encontró congestión del hígado, pulmón, riñón, bazo y tráquea, hemorragia pulmonar, gastritis con desprendimiento de mucosa, hemorragia en el endocardio y edema cerebral. Hemopericardio se observó en un animal. Estas lesiones patológicas se atribuyen a la acción tóxica de las proteínas de los macroquistes y coinciden con las descritas por Sam et al. (1998).

La altas temperaturas de los métodos de cocción, horneado y fritura fueron efectivos en reducir la letalidad de la toxina (Durán, 2004; Céspedes, 2005). Este efecto se atribuye al aumento de la energía cinética de las moléculas con lo que se desorganiza la envoltura acuosa de las proteínas y se desnaturalizan (Lesk, 2001). Es decir, un aumento de la temperatura destruye las interacciones débiles y desorganiza la estructura de la proteína, de forma que el interior hidrofóbico interacciona con el medio acuoso y se produce la agregación y precipitación de la proteína desnaturalizada (Girard, 1991; Prändl et al., 1994).

El método de congelación no logró inactivar completamente la toxina aunque pudo evitar la muerte de los animales. La acción de la congelación se atribuye a la formación de cristales de hielo, los cuales extraen agua ligada a las proteínas, de tal forma que se desorganizan (Jasper y Placzek, 1978; Lawrie, 1998). De otra parte, los mayores daños celulares se dan en una congelación lenta y a temperaturas bajas (hasta $-10{ }^{\circ} \mathrm{C}$ ) (Prändl et al., 1994; Warris, 2003).

El examen coprológico mostró que sólo los perros del grupo control positivo eliminaron esporoquistes a partir de los 14 días de la ingestión de carne no tratada, confirmando el efecto benéfico de la cocción, horneado, congelado y frito. El efecto de los tres primeros métodos en carne de CSA ha sido reportado por otros autores (Gorman et al., 1984; Leguía y Arévalo, 1990; Céspedes, 2005), pero éste es el primer reporte usando la fritura. Otros trabajos mencionan el efecto de la temperatura sobre la carne de cerdo (Fayer, 2004).

\section{Conclusiones}

- La cocción, horneado y fritura lograron desnaturalizar y detoxificar la sustancia proteica, sarcocystina, de los macroquistes de Sarcocystis aucheniae.

- El congelamiento sólo pudo eliminar parcialmente la letalidad pero no la toxicidad de la sarcosystina.

- La ingestión de carne de llama tratada con cocción, horneado, fritura y congelación afectó la viabilidad de los quistes, eliminando el riesgo potencial de infección del perro.

\section{Literatura Citada}

1. Alva, J.; M. Rojas; A. Nuñez. 1980. Decomisos por parasitosis y su importancia económica en alpacas (Lama pacos). Rev. Inv. Pec. IVITA 5(1): 61-62.

2. Castro, J. 1974. Sarcocystis aucheniae en llamas (Lama glama). Rev. Inv. Pec. IVITA 3(1): 91-92.

3. Céspedes, C. 2005. Saneamiento y detoxificación de la carne de alpaca con sarcocistiosis mediante tratamientos físicos y químicos (marinado y salazón) de uso doméstico. Tesis de Médico Veterinario. Facultad de Medicina Veterinaria, Univ. Nacional Mayor de San Marcos. Lima. 75 p.

4. Cordero del Campillo, M. 1999. Parasitología veterinaria. McGraw-Hill Interamericana. España. p 319-328.

5. Durán, J. 2004. Saneamiento y detoxificación de la carne de alpaca con Sarcocystis mediante la aplicación de tratamientos físico-químicos apropiados 
para uso doméstico. Tesis de Médico Veterinario. Facultad de Medicina Veterinaria, Univ. Nacional Mayor de San Marcos. Lima. 54 p.

6. Fayer, R. 2004. Sarcocystis spp. in human infections. Clin. Microbiol. Rev. 17: 894-902.

7. Girard, J.P. 1991. Tecnología de la carne y de los productos cárnicos. Ed. Acribia. Zaragoza, España. 250 p.

8. Gorman, T.; H. Alicaino; H. Muñoz. 1984. Sarcocystis sp. in guanaco (Lama guanicoe) and effect of temperatura on its viability. Vet. Parasitol. 15: 95-101.

9. Guerrero, C; J. Hernández; J. Alva. 1967. Sarcocystis en alpacas. Rev. Fac. Med. Lima. 21: 69-76.

10. Guinart, N.; J. López. 1997. ¿Qué sabemos de la fiebre?. Rev. Cubana Med. Gen. Integr. 13 (2) [Internet]. [acceso 10 junio 2006]. Disponible en: http:// www.bvs.sld.cu/revistas/mgi/ vol13_2_97/mgi09297.htm

11. Hiepe, F; L. Lietzke; G. Scheiber; R. Jungmann; T. Hiepe; T. Montag. 1981. Untersuchungen zur toxischen wirkung von extrankten aus Sarcocystis ovifelis macrocysten auf Kanichen. Mh. Vet. Med. 36: 908-910.

12. Jasper, W.; R. Placzek. 1978. Conversación de la carne por el frío. Ed. Acribia. Zaragoza, España.

13. Lawrie, R. 1998. Ciencia de la carne. $3^{\text {ra }}$ ed. p 187-281. Ed. Acribia. Zaragoza, España. 131 p.

14. Leguía, G. 1987. Enfermedades infecciosas y parasitarias de las alpacas. CICCS-UNMSM-IVITA. p $38-43$.

15. Leguía, G. 1991. The epidemiology and economic impact of llama parasites. Parasitol. Today 7: 54-56.

16. Leguía, G. 1999. Enfermedades parasitarias y atlas parasitológico de camélidos sudamericanos. Ed. De Mar. Lima. p 23-29.

17. Leguía, G.; C. Guerrero; R. Sam; A. Chávez. 1989. Infección experimental de perros y gatos con micro y macroquistes de Sarcocystis de alpacas (Lama pacos). MV Rev. Cienc. Vet. Lima 5(3): 10-13.

18. Leguía, G.; N. Clavo. 1989. Sarcocystis o "triquina". Bol. Div. No 7. IVITA-UNMSM-CICCS. p 12-14.

19. Leguía, G.; F. Arévalo. 1990. Efecto de la cocción, refrigeración, congelación y deshidratación (charqui) sobre la viabilidad de Sarcocystis de alpacas. MV Rev. Cienc. Vet. Lima 6(1): 19-20.

20. Lesk, A. 2001. Introduction to protein architecture. The structural biology of proteins. Oxford University Press New York.

21. Medrano, G. 2006. Estudio filogenético de Sarcocystis basado en el análisis del gen SSU rRNA. Estudio ultraestructiural y detección molecular temprana en alpacas del Perú. Tesis de Doctor en Ciencias. Univ. Peruana Cayetano Heredia. Lima. $131 \mathrm{p}$.

22. Prändl, O.; A, Fischer; T. Schmidhorfer; H. Sinell. 1994. Tecnología e higiene de la carne. Ed. Acribia. España. p 237-302.

23. Sam, R. 1988. Sarcocystis aucheniae: Caracterización parcial de componentes antigénicos y patología clínica experimental en alpacas. Tesis de Doctor en Ciencias Biológicas. Univ. Nacional Mayor de San Marcos. Lima. 118 p.

24. Sam, R.; I. Mansilla; C. Morales; A. Ramírez. 1998. Efecto tóxico de macroquistes de Sarcocystis aucheniae en ratones, cobayos y conejos. Rev. Inv. Pec. IVITA 9(2): 11-18.

25. Snedecor, G.; W. Cochran. 1989. Statistical methods. $8^{\text {h }}$ ed. Iowa State University Press. Iowa, USA. 503 p.

26. Soulsby, E. 1988. Parasitología y enfermedades parasitarias en los animales domésticos. $7^{\mathrm{a}}$ ed. p 681-697. Ed. Interamericana. México.

27. Warris, $\boldsymbol{P}$. 2003. Ciencia de la carne. Ed. Acribia. Zaragoza, España. p 204212. 